\title{
Work at FNAL to achieve long Electron Drift Lifetime in Liquid Argon
}

\author{
D. Finley, W. Jaskierny, C. Kendziora, J. Krider, \\ S. Pordes, P.A. Rapidis, T. Tope \\ Particle Physics Division, Fermilab
}

October 27, 2006

\section{Introduction}

This note records some of the work done between July 2005 and July 2006 to achieve long (many milliseconds) electron drift lifetimes in liquid argon at Fermilab [1]. The work is part of a process to develop some experience at Fermilab with the technology required to construct a large liquid argon TPC. This technology has been largely developed by the ICARUS collaboration in Europe and this process can be seen as technology transfer. The capability to produce liquid argon in which electrons have drift lifetimes of several milliseconds is crucial to a successful device. Liquid argon calorimeters have been successfully operated at Fermilab; their electro-negative contaminants are at the level of $10^{-7}$ while the TPC we are considering requires a contamination level at the level of $10^{-11}$, tens of parts per trillion (ppt). As well as demonstrating the ability to produce liquid argon at this level of purity, the work is part of a program to test the effect on the electron drift time of candidate materials for the construction of a TPC in liquid argon.

\section{General Description}

Both the scheme adopted to achieve clean argon and the technique used to measure the electron drift lifetime were copied from ICARUS [2]. That the oxygen filter should operate on liquid argon was an ICARUS development [3], and the device used to measure the lifetime, the so-called Purity Monitor or PrM [4], is also an ICARUS development. Two things are new in our implementation; one is the oxygen filter material used, a copper alumina catalyst [5], and the second is that the filter can be and has been regenerated in place. The FNAL purity monitors also differed from the version we were loaned in some details. The long rods were made from PEEK [7] instead of G-10. To reduce electrical noise, the internal cabling was changed from RG-316 to RG-180 and the internal

*now at Demokritos Institute, Athens, Greece 
connection topology of the cable shields was altered to improve the current return. We also used RG-180 cable, because of its low capacitance, for the external signals [10].

The figure below is a schematic of the liquid argon system. Some key features are (following the liquid argon flow): the molecular sieve used to trap water vapor [6] with the capability for bake-out, the filter for oxygen with the capability for bake-out and regeneration, valving to allow any section of the input line to be purged and/or evacuated, pressure reliefs all over the place, and a switch to allow the argon to be diverted just before entering the cryostat. As can be seen, the argon lines run in vacuum. The cryostat and the vacuum insulation space for the argon lines had separate pumping systems.

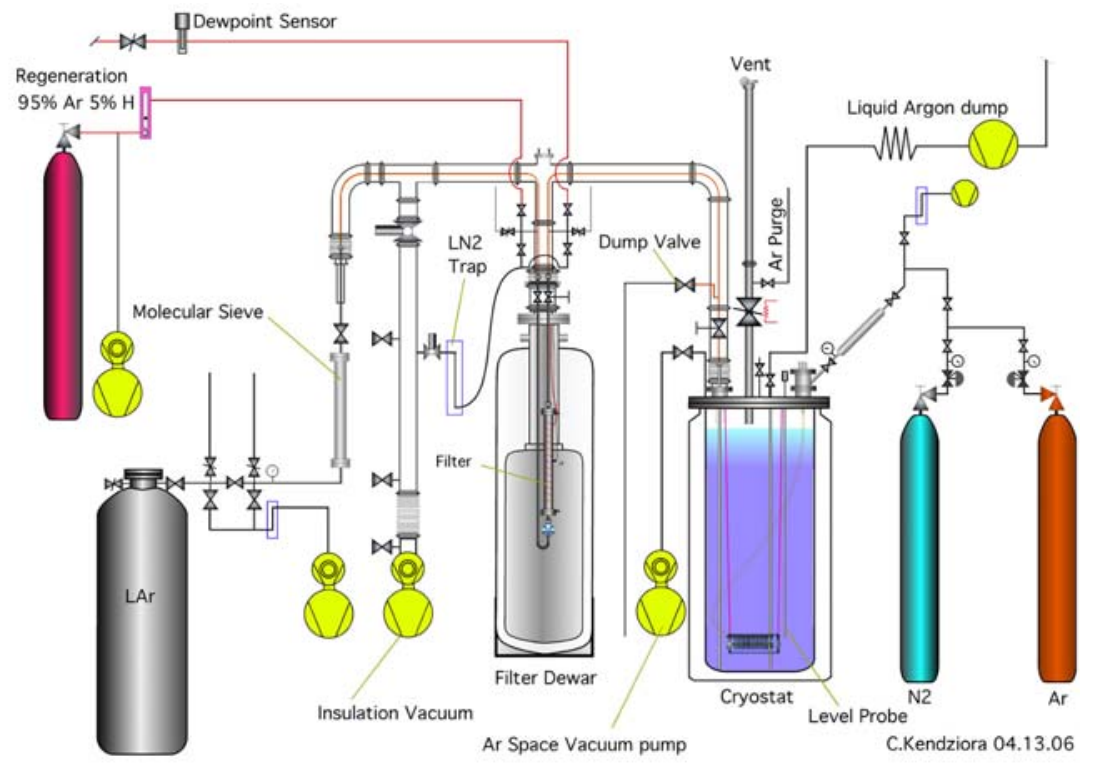

Apart from the purity monitor, the cryostat itself was equipped with a system to inject gas (such as Nitrogen) to test the effect on the lifetime, pressure gauges, and a commercial liquid level indicator. The argon system was single pass since the cryostat available [8] was only rated for a few inches of water overpressure.

Since there was no way to recirculate the argon through a filter and measurements on a single fill could spread over several days, the system was extremely susceptible to leaks, including any permeation through O-ring seals [9]. All seals in the argon system were metal except for the top flange of the cryostat which used a double o-ring with an argon purge applied between the two rings. The typical vacuum achieved before filling with argon was a few $10^{-6}$ where the purity monitor and associated cabling were the major source of gas, presumably water-vapor. The next figure is a photograph of a version of the setup in the PAB (Proton Assembly Building) before the gas injection system was added. 


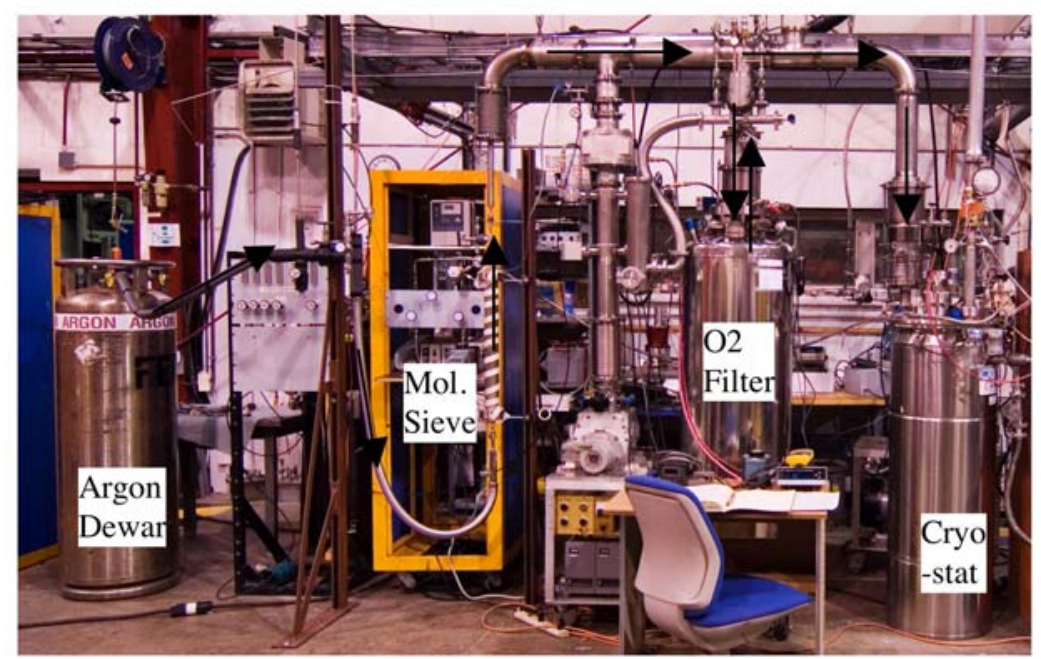

\section{Procedure}

A new run involved a bake-out of the molecular sieve and a regeneration of the oxygen filter following the instructions from the respective manufacturers. Heaters, thermocouples and controls were implemented for this. A dew-point monitor in the exhaust stream from the oxygen filter was installed to indicate that the regeneration was complete. After baking and regeneration, the input argon system was maintained under argon. A commercial grade dewar of liquid argon (few ppm of Oxygen) would be connected at the input, the input line upstream of the molecular sieve was purged and argon would be allowed to flow through the system diverting the output just before the cryostat. When liquid appeared at this output, the cryostat was isolated from its vacuum pump, the diversion outlet closed and argon allowed to enter the cryostat. This procedure was adopted with the idea that any residual contaminants would be removed by the initial argon flow.

It took two commercial dewars to fill the cryostat, in a total time of about 1.5 hrs. The procedure for removing the empty dewar and attaching the new one simply involved closing the input to the cryostat, purging the input line with the new dewar and then allowing flow into the cryostat.

\section{Read-out and Data Taking}

The electronics is based on the DZero calorimeter integrator; details of the electronics and wiring can be found in [10]. We read the cathode and anode signals with separate sets of electronics. This necessitates a cross calibration of the electronics for each channel - the difference in gain was less than a few \% but has the advantage of allowing us to investigate systematics due to different grid material geometries. The light source used is a Hamamatsu Xenon flash lamp [11]; a non-solarizable UV quartz fiber [12] carries the light to illuminate a gold photocathode [13]. The electron drift life-time can be determined from the 
size of the cathode signal, the size of the anode signal and the time between the two signals. The outputs of the anode and cathode integrators were connected to a digital oscilloscope [14] and the numbers taken from the display by hand. Because the fall time of our integrators was rather fast, the cathode signal peak is an underestimate of the cathode charge. We therefore fixed the cathode field (and thus the charge) and set various values of the drift field - thus producing different drift times - and infer the drift lifetime from a plot of $\log _{e}$ (Anode Signal/Cathode Signal) vs time. The original purity monitor has a drift length of about $17 \mathrm{cms}$. We have also made and used a $50 \mathrm{~cm}$ version, shown below.

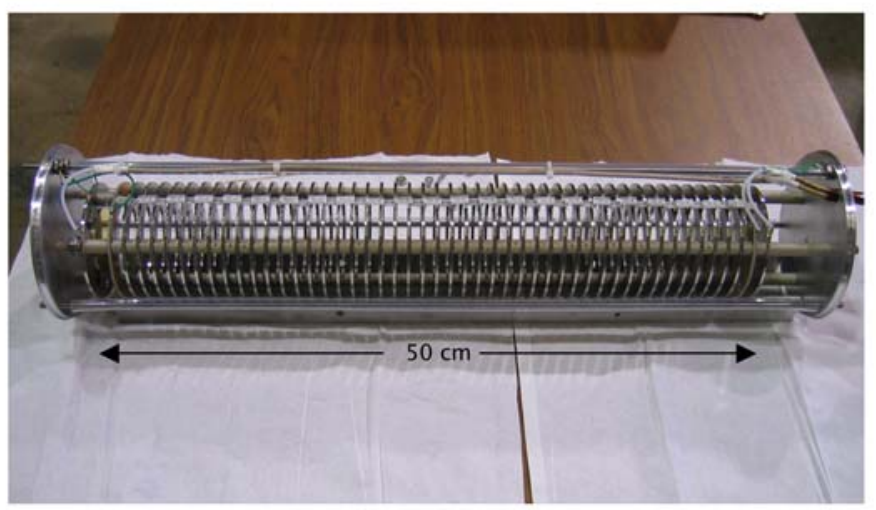

Figure 4 shows a scope display where the cathode and anode signals are separated by 5.7 milliseconds. The fall-time of the integrator is small compared to the drift time so the signals look like pulses rather than steps. At long drift times, the signals are small because the electric fields applied are low and the yield of photoelectrons depends on the electric field at the cathode.

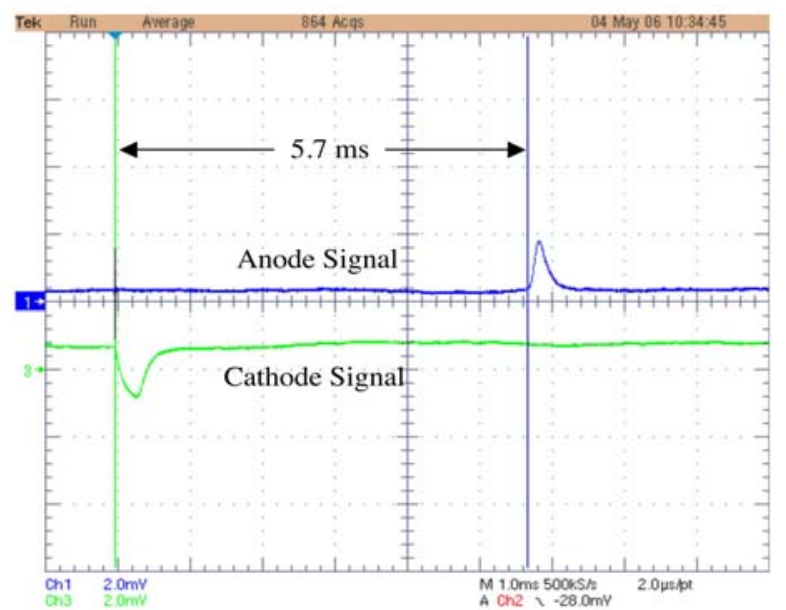


A set of traces collected with fixed cathode field and different drift fields is shown below.

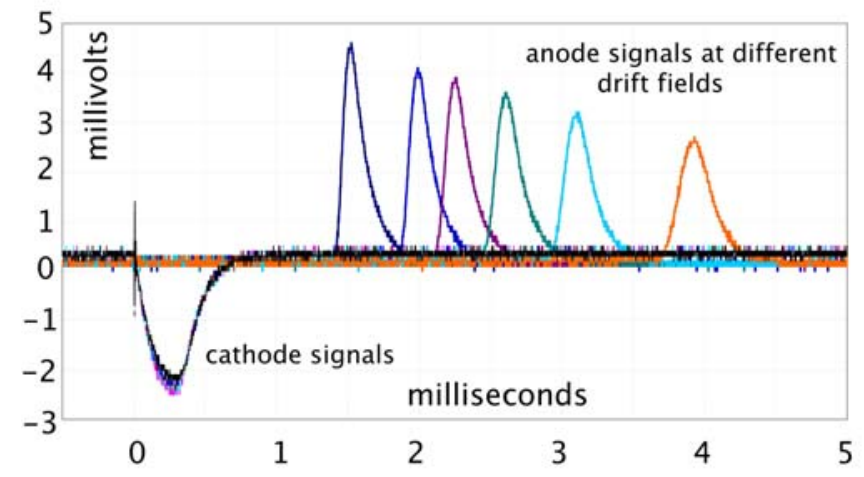

These data give a typical plot made to infer the drift lifetime as shown next. The slope of the plot gives the lifetime, in this case $1 / 0.135, \sim 7.4$ milliseconds.

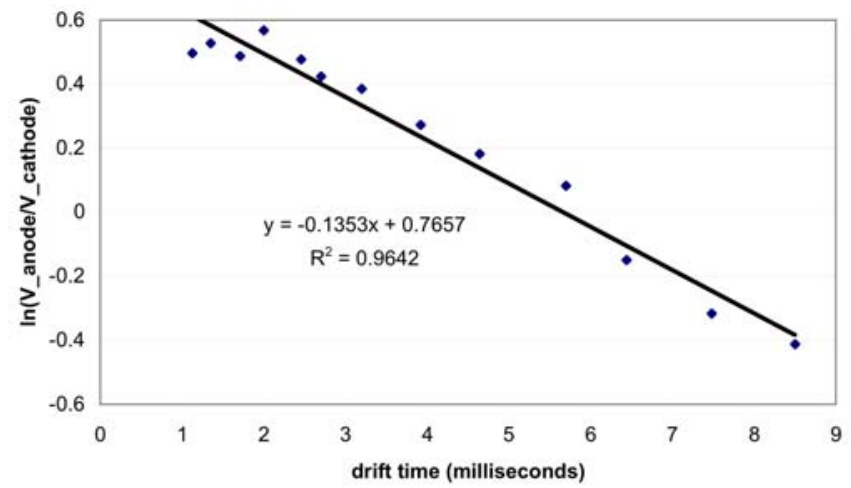

The data also give the drift velocity as a function of electric field.

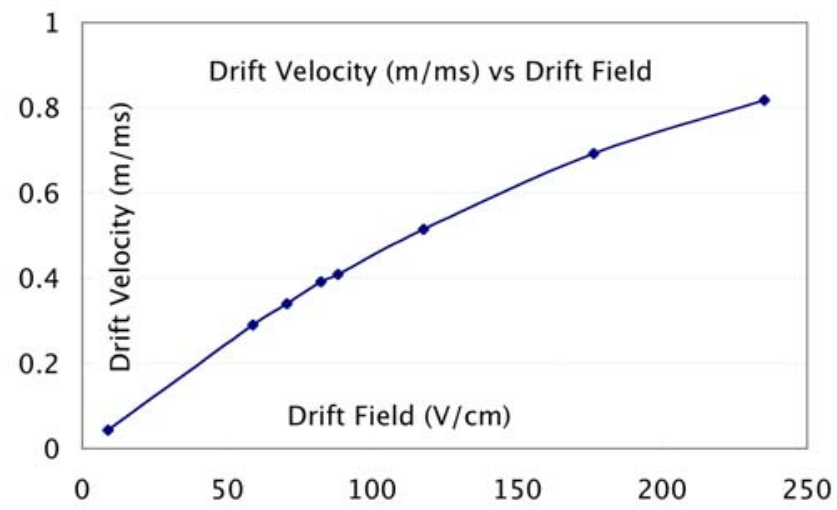




\section{Anecdotes}

Two issues attracted our attention and though they have not been resolved systematically may be worth noting. One is that water vapor in the liquid argon seems to affect the drift lifetime achieved. Two observations support this statement. One is that before the molecular sieve was implemented, we could improve the lifetime achieved on successive fills by baking - but not regenerating - the oxygen filter. It is known that the oxygen filter will absorb water-vapor. We do not know if the improvement in lifetime after baking is because the baking removes water-vapor in the filter which affects the filter's ability to remove oxygen or because after baking the filter can now trap the water-vapor which affects the drift lifetime. The second observation is that before the molecular sieve was implemented, the drift lifetime would deteriorate rapidly after about $1 / 2$ a dewar of argon had passed into the cryostat. The combination of these observations suggests an effect on the oxygen filter but this is not conclusive.

A second item we note is that the drift lifetime of a given load of argon tended to improve with time. In particular there was a striking improvement over a few hours just after the fill. The figure below shows the ratio of anode to cathode signal at a fixed drift time over a few days. This feature has been observed in other liquid argon systems and may be due to electro-negative impurities coating out of the liquid onto the walls of the cryostat. This is possibly a concern for a large volume device where this sort of improvement may be reduced since the surface to volume ratio is lower.

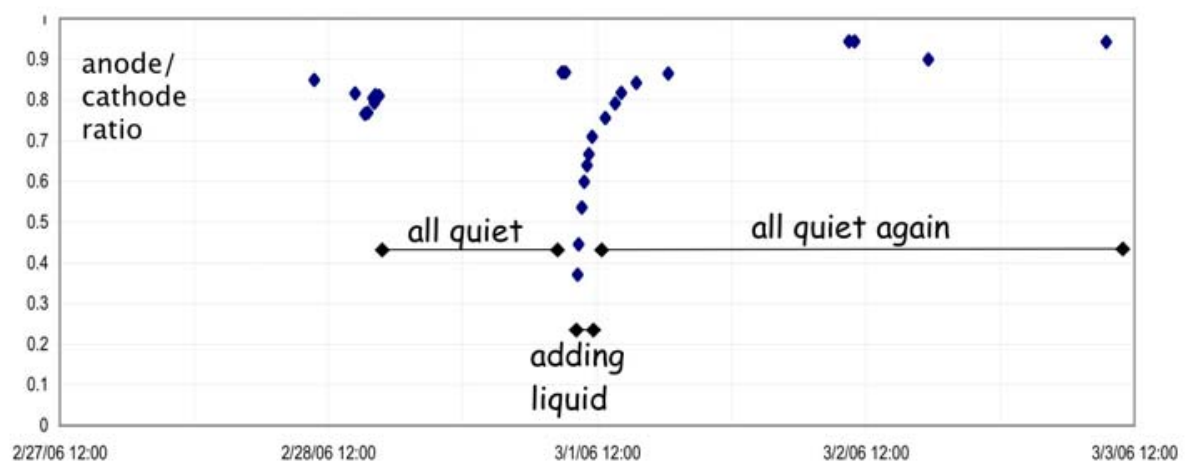

Table of Volumes and Flow rates[15]

\begin{tabular}{|lr|}
\hline Commercial Liquid Argon Dewar & 165 liters (230 kg) \\
MSU Test Cryostat & 178 liters, 145 liters fill \\
Molecular Sieve of Sigma-Aldrich 5A & 1.72 liters, 1212 gms \\
$\mathrm{O}_{2}$ Filter of Engelhard CU-0226S & 1.72 liters, 1393 gms \\
Flow Rate & $1 \mathrm{LPM}$ \\
Fills between Regeneration & 5 \\
\hline \hline
\end{tabular}


We hope soon to start commissioning the Material Test Station. This facility will allow us to introduce materials into the liquid argon and observe their effect on the drift lifetime. A materials carriage allows the material to be lowered into the argon without otherwise disturbing the system. The material can be purged with argon before being inserted. A schematic and a photograph of the MTS are shown below.

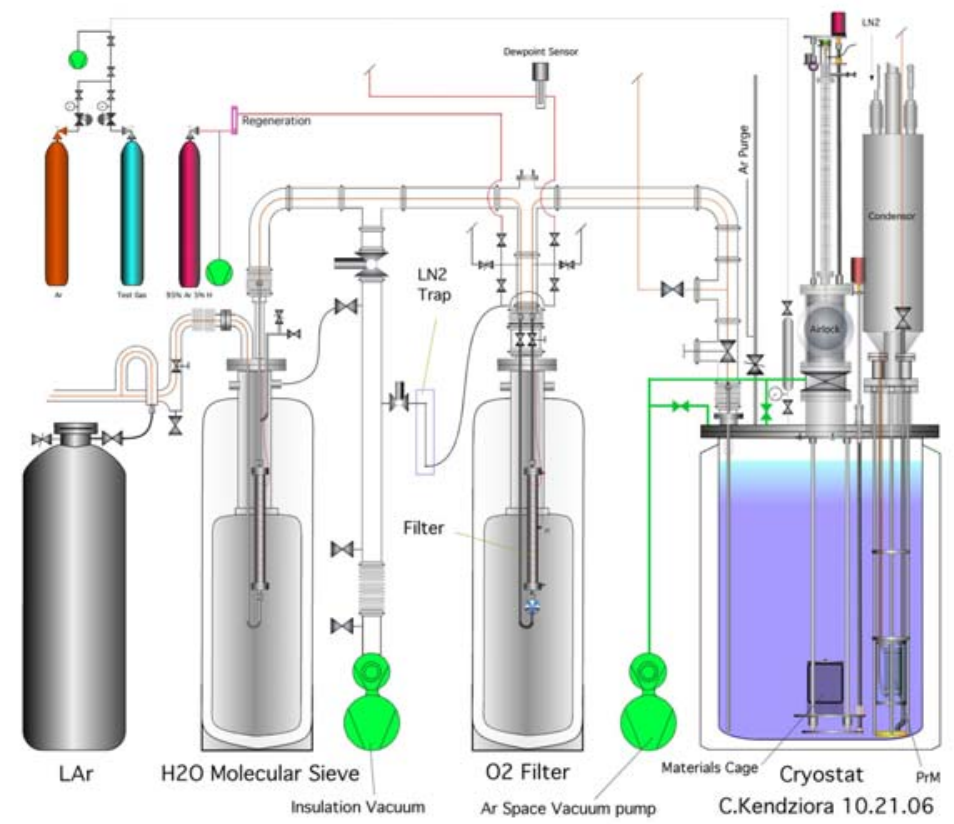

The Material Test System as of 10/06

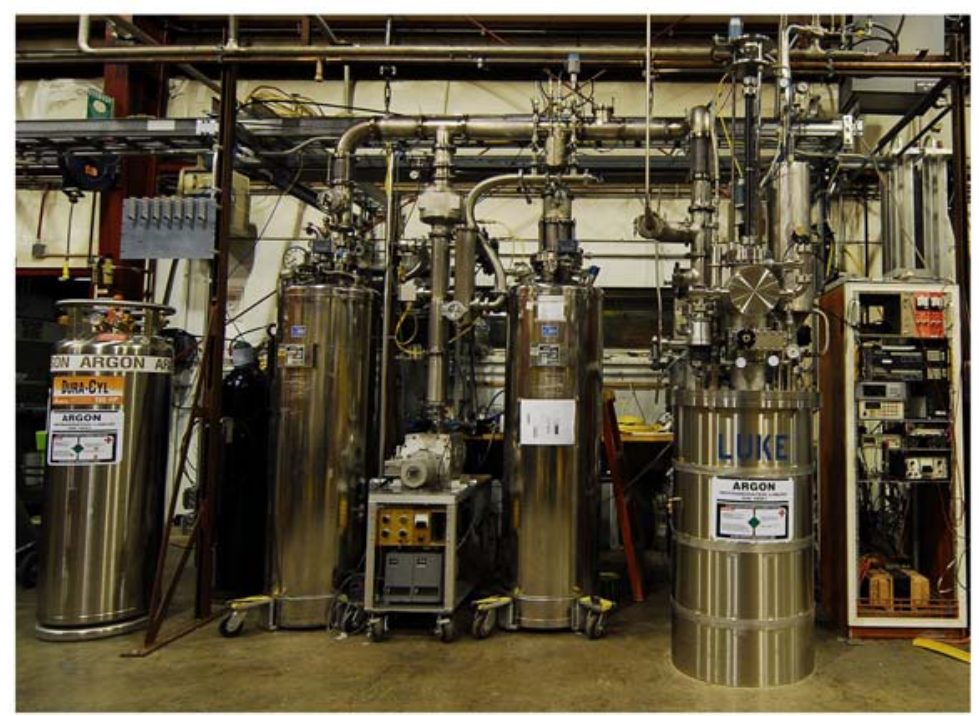




\section{References}

[1] The motivation to develop Liquid Argon TPC's at Fermilab comes from Adam Para.

[2] ICARUS Collaboration, Nucl. Instrum. Meth. A 527329 (2004).

[3] P. Benetti et al., Nucl. Instrum. Meth. A 333, 567 (1993).

[4] G. Carugno et al., Nucl. Instrum. Meth. A 292580 (1990).

[5] Engelhard Copper Alumina catalyst CU-0226S

[6] Sigma-Aldrich 5A material

[7] PEEK, Polyetheretherketone.

[8] The cryostat was kindly loaned to us by Michigan State University.

[9] T. Tope, 'Estimate of oxygen permeation thru o-rings in test setup' in http://lartpc-docdb.fnal.gov/cgi-bin/ShowDocument?docid=38

[10] W. Jaskierny, Schematics of the circuit and connections in http://lartpc-docdb.fnal.gov/cgi-bin/ShowDocument?docid=32

[11] Hamamatsu L7685 with C6096 Power supply rated at 1 joule.

[12] Solarization Resistant Optical Fiber, 600 micron Diameter, Part Number 2001525, Polymicro, Phoenix, Az 85023.

[13] The photocathodes used here were made at Fermilab in the Vacuum Deposition Facility. The picture below shows the belljar setup where we test candidate photocathode materials in vacuum and in argon, and observe their behavior over time.

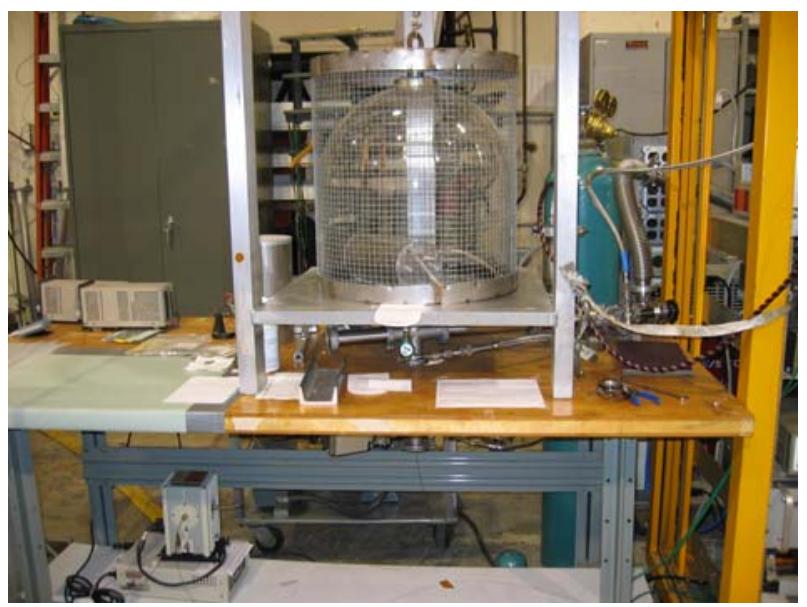


[14] 4 channel 300 MHZ bandwidth 1 Gigasample/second Tektronix TDS 5034.

[15] Further details are in T. Tope http://lartpc-docdb.fnal.gov/cgi-bin/ShowDocument?docid=91 